\title{
$p$-DUAL FRAMES AND $p$-RIESZ SEQUENCES IN QUASINORMED SPACES
}

\author{
JOSÉ ALFONSO LÓPEZ NICOLÁS
}

\begin{abstract}
The present contribution is aimed at obtaining new results in duality between $p$-dual frames and $p$-Riesz sequences in quasinormed spaces with a normalized Schauder basis. We obtain two results which show the relationship of duality between these concepts. We split a quasinormed space into a topological sum of two subspaces and use the Schauder basis to establish a relationship between the $p$-dual frames of one of these subspaces and the $p$-Riesz sequences of the dual of the other one. In fact, the main results are stated in a lightly more general context.
\end{abstract}

\section{INTRODUCTION}

In 1946 Gabor introduced a fundamental approach to signal decomposition in terms of elementary signals (see [8]). In 1952 Duffin and Schaeffer abstracted the work of Gabor to define frames for a Hilbert space ([7]). In 1986 the work of Daubechies, Grossman, and Meyer initiated the theory of wavelets ([6]).

In 1991 Gröchenig defined Banach frames for a Banach space $X$ with respect to an associated Banach space $X_{d}$ of scalar valued sequences indexed by $\mathbb{N}$ :

Definition 1.1 (Gröchenig, [9]). Let $X$ be a Banach space and let $X_{d}$ be an associated Banach space of scalar valued sequences indexed by $\mathbb{N}$. Let $\left(y_{i}\right)_{i \in \mathbb{N}}$ be a sequence of elements from $X^{*}$ and let $S: X_{d} \rightarrow X$ be given. If

(1) $\left(y_{i}(x):=\left\langle x, y_{i}\right\rangle\right)_{i \in \mathbb{N}} \in X_{d}$, for each $x \in X$,

(2) the norms $\|x\|_{X}$ and $\left\|\left(\left\langle x, y_{i}\right\rangle\right)_{i \in \mathbb{N}}\right\|_{X_{d}}$ are equivalent,

(3) $S$ is bounded and linear, and $S\left(\left\langle x, y_{i}\right\rangle\right)_{i \in \mathbb{N}}=x$, for each $x \in X$,

then $\left(\left(y_{i}\right)_{i \in \mathbb{N}}, S\right)$ is a Banach frame for $X$ with respect to $X_{d}$. The mapping $S$ is the reconstruction operator. If the norm equivalence is given by

$$
A\|x\|_{X} \leq\left\|\left(\left\langle x, y_{i}\right\rangle\right)_{i \in \mathbb{N}}\right\|_{X_{d}} \leq B\|x\|_{X}
$$

then $A, B$ are a choice of frame bounds for $\left(\left(y_{i}\right)_{i \in \mathbb{N}}, S\right)$.

In 1999, Casazza, Han, and Larson defined frames and normalized tight frames for Banach spaces.

2010 Mathematics Subject Classification. 46B25, 46E99, 46B20.

Key words and phrases. Quasinormed spaces; Banach spaces; dual space; $p$-dual frame; $p$-Riesz sequence; Schauder basis. 
Definition $1.2\left(\left[3\right.\right.$, Definition 3.3]). A sequence $\left(x_{i}\right)_{i \in \mathbb{N}}$ in a Banach space $\mathrm{X}$ is a frame for $X$ if there is a Banach space $Z$ with an unconditional basis $\left(z_{i}, z_{i}^{*}\right)$ with $X \subset Z$ and a (onto) projection $P: Z \rightarrow X$ so that $P z_{i}=x_{i}$ for all $i \in \mathbb{N}$. If $\left(z_{i}\right)$ is a 1 -unconditional basis for $Z$ and $\|P\|=1$, we will call $\left(x_{i}\right)$ a normalized tight frame for $X$.

In 2000 Aldroubi, Sun, and Tang defined the concept of $p$-frame for a normed linear space, with $1 \leq p \leq \infty$ (see [1]).

Definition 1.3 ( $p$-frame, [1]). Let $1 \leq p \leq \infty, X$ be a normed linear space, and $X^{*}$ its dual. We say that an index family $\left\{g_{\lambda}: \lambda \in \Lambda\right\} \subset X^{*}$ is a $p$-frame for $X$ if there exists a positive constant $C$ such that

$$
C^{-1}\|f\|_{X} \leq\left\|\left\{\left(f, g_{\lambda}\right)\right\}_{\lambda \in \Lambda}\right\|_{p} \leq C\|f\|_{X}, \quad \forall f \in X
$$

In this paper we will define the concept of $p$-dual frame for quasinormed linear spaces, which is dual to their concept of $p$-frame. They also used in [1] the concept of $p$-Riesz basis for $L^{p}$, with $1 \leq p \leq \infty$.

Definition 1.4 ( $p$-Riesz basis, [1]). Let $1 \leq p \leq \infty$. We say that a countable collection $\left\{g_{\lambda}: \lambda \in \Lambda\right\} \subset L^{p}$ is a $p$-Riesz basis for $L^{p}$ if there exists a positive constant $C$ such that

$$
C^{-1}\|c\|_{p} \leq\left\|\sum_{\lambda \in \Lambda} c_{\lambda} g_{\lambda}\right\|_{p} \leq C\|c\|_{c}, \quad \forall c=\left(c_{\lambda}\right)_{\lambda \in \Lambda} \in l^{p}(\Lambda) .
$$

In addition, they defined in [1] the locally finite shift invariant subspaces $V_{p}(\varphi)$ of $L^{p}$, and obtained a connection between certain $p$-frames and $p$-Riesz bases of those spaces. The authors continued this study in [2] (2001).

In 2005 Casazza, Christensen, and Stoeva defined in [4] the BK-spaces $X_{d}$, which are Banach sequence spaces whose coordinate functionals are continuous, and also defined $X_{d}$-frames, generalizing $p$-frames (see Definition 1.3 ).

Definition 1.5 ([4, Definition 1.2]). Let $X$ be a Banach space and $X_{d}$ a BK-space. A countable family $\left\{g_{i}\right\}_{i \in I}$ in the dual $X^{*}$ is called an $X_{d^{-}}$-frame for $X$ if

(1) $\left\{g_{i}(f)\right\}_{i \in I} \in X_{d}, \forall f \in X$;

(2) the norms $\|f\|_{X}$ and $\left\|\left\{g_{i}(f)\right\}_{i \in I}\right\|_{X_{d}}$ are equivalent, i.e., there exist constants $A, B>0$ such that $A\|f\|_{X} \leq\left\|\left\{g_{i}(f)\right\}_{i \in I}\right\|_{X_{d}} \leq B\|f\|_{X}$.

$A$ and $B$ are called $X_{d}$-frame bounds. If at least (1) and the upper condition in (2) are satisfied, $\left\{g_{i}\right\}_{i \in I}$ is called an $X_{d}$-Bessel sequence for $X$.

If $X$ is a Hilbert space and $X_{d}=l^{2}$, (2) means that $\left\{g_{i}\right\}_{i \in I}$ is a frame. If $X_{d}=l^{p}$, with $1 \leq p \leq \infty$, then $X_{d}$-frames for $X$ are exactly the $p$-frames for $X$, by Definition 1.3 .

These authors also investigated the existence of $X_{d}$-frames and Banach frames in separable Banach spaces, and revealed the connections between Banach frames and the reconstruction property (see [4]). 
In 2009 Stoeva studied $X_{d}$-frames for Banach spaces and defined the dual and dual $^{*}$ of an $X_{d}$-Bessel sequence. She obtained a connection between both concepts and conditions for their existence (see [13]).

In 2015 Olevskii and Ulanovskii established a duality relationship between frames and Riesz sequences in Hilbert spaces in their research on stable sampling and interpolation theory.

Theorem 1.6 ([11]; [12, p. 10]). Assume that a set $U$ is an orthonormal basis in a Hilbert space $H$. Assume that $H$ is a direct sum of two orthogonal subspaces $H_{1}$ and $\mathrm{H}_{2}$, and denote by $P_{j}$ the orthogonal projection on $H_{j}$. Assume further that $U$ is a union of two disjoint subsets $V$ and $W$. Then the following statements are equivalent:

(1) $P_{1}(V)$ is a frame in $H_{1}$.

(2) $P_{2}(W)$ is a Riesz sequence in $H_{2}$.

The objective of this paper is to generalize this last theorem to quasinormed spaces. In this general context we have no inner product, so we will need additional hypotheses for replacing the orthogonality conditions.

1.1. Our definitions. In this paper we work with the concepts of $p$-dual frame, in a dual sense to Definition 1.3 , and $p$-Riesz sequence for quasinormed spaces and $p \in(0,+\infty]$. We also work with the concept of a Schauder basis of a quasinormed space. Next we establish the definitions of all these concepts. Given a set $I$, we denote by $\mathfrak{P}_{0}(I)$ the set of all finite subsets of $I$.

Definition 1.7. Let $(E,\|\|)$ be a quasinormed space. Let $\mathcal{B}:=\left(u_{i}\right)_{i \in \mathbb{N}}$ be a sequence in $E$.

$(1) \mathcal{B}$ is called a generator set for $(E,\|\|)$ if for every $x \in E$ there exists a sequence $c_{x}=\left(c_{i}\right)_{i \in \mathbb{N}}$ of complex numbers such that the series $\sum_{i \in \mathbb{N}} c_{i} u_{i}$ converges to $x$.

$(2) \mathcal{B}$ is a Schauder basis for $(E,\|\|)$ if for every $x \in E$ there exists a unique sequence $c_{x}=\left(c_{i}\right)_{i \in \mathbb{N}}$ of complex numbers such that the series $\sum_{i \in \mathbb{N}} c_{i} u_{i}$ converges to $x$.

(3) $\mathcal{B}$ is said to be normalized if $\left\|u_{i}\right\|=1$ for each $i \in \mathbb{N}$.

Definition 1.8 ( $p$-Riesz sequence). Let $(E,\|\|)$ be a quasinormed space and $p \in$ $(0,+\infty]$. Let $\left(u_{i}\right)_{i \in \mathbb{N}}$ be a sequence in $E$.

(1) $\left(u_{i}\right)_{i \in \mathbb{N}}$ is an upper $p$-Riesz sequence for $(E,\|\|)$ if there exists a constant $A>0$ such that for every $I_{0} \in \mathfrak{P}_{0}(\mathbb{N})$ and every $\left(c_{i}\right)_{i \in I_{0}} \in \mathbb{C}^{I_{0}}$ we have that

$$
A\left\|\left(c_{i}\right)_{i \in I_{0}}\right\|_{p} \leq\left\|\sum_{i \in I_{0}} c_{i} u_{i}\right\| .
$$

In that case the constant $A$ is called a constant of upper $p$-Riesz sequence of $\left(u_{i}\right)_{i \in \mathbb{N}}$ for $(E,\|\|)$. 
(2) $\left(u_{i}\right)_{i \in \mathbb{N}}$ is a lower $p$-Riesz sequence for $(E,\|\|)$ if there exists a constant $B>0$ such that for every $I_{0} \in \mathfrak{P}_{0}(\mathbb{N})$ and every $\left(c_{i}\right)_{i \in I_{0}} \in \mathbb{C}^{I_{0}}$ we have that

$$
\left\|\sum_{i \in I_{0}} c_{i} u_{i}\right\| \leq B\left\|\left(c_{i}\right)_{i \in I_{0}}\right\|_{p} .
$$

In that case the constant $B$ is called a constant of lower $p$-Riesz sequence of $\left(u_{i}\right)_{i \in \mathbb{N}}$ for $(E,\|\|)$.

(3) $\left(u_{i}\right)_{i \in \mathbb{N}}$ is a $p$-Riesz sequence for $(E,\|\|)$ if it is both upper and lower $p$-Riesz sequence for $(E,\|\|)$.

Definition 1.9 ( $p$-Dual frame). Let $(E,\|\|)$ be a quasinormed space and $p \in$ $(0,+\infty]$. Let $\left(u_{i}\right)_{i \in \mathbb{N}}$ be a sequence in $E$.

(1) $\left(u_{i}\right)_{i \in \mathbb{N}}$ is a lower $p$-dual frame (briefly, lower $p$-frame* for $^{*}(E,\|\|)$ if there exists a constant $A>0$ such that for each $f \in E^{*}$ we have that

$$
A\|f\|_{*} \leq\left\|\left(f\left(u_{i}\right)\right)_{i \in \mathbb{N}}\right\|_{p}<\infty .
$$

In that case the constant $A$ is called a constant of lower $p$-dual frame of $\left(u_{i}\right)_{i \in \mathbb{N}}$ for $(E,\|\|)$.

(2) $\left(u_{i}\right)_{i \in \mathbb{N}}$ is an upper $p$-dual frame (briefly, upper $p$-frame* ${ }^{*}$ for $(E,\|\|)$ if there exists a constant $B>0$ such that for each $f \in E^{*}$ we have that

$$
\left\|\left(f\left(u_{i}\right)\right)_{i \in \mathbb{N}}\right\|_{p} \leq B\|f\|_{*} .
$$

In that case the constant $B$ is called a constant of upper $p$-dual frame of $\left(u_{i}\right)_{i \in \mathbb{N}}$ for $(E,\|\|)$. An upper $p$-dual frame is also called a $p$-dual Bessel sequence.

(3) $\left(u_{i}\right)_{i \in \mathbb{N}}$ is a $p$-dual frame (briefly, $p$-frame*) for $(E,\|\|)$ if it is both upper $p$-dual frame and lower $p$-dual frame for $(E,\|\|)$. In other words, the dual norm \|\|$_{*}$ is equivalent to the one defined by $\|f\|_{*, p}:=\left\|\left(f\left(u_{i}\right)\right)_{i \in \mathbb{N}}\right\|_{p}$ for each $f \in E^{*}$.

Observe that for normed spaces the concept of $p$-frame* is dual to that of $p$-frame (see Definition 1.3. For Hilbert spaces both concepts are equivalent.

1.2. Our results. In this paper we work with quasinormed spaces with a normalized Schauder basis (in fact, in a more general setting). We split these spaces into a topological direct sum of two subspaces and we investigate conditions to determine the relationship between the $p$-dual frames of one of these subspaces and the Riesz $p$-sequences of the dual space of the other one, with $p \in(0,+\infty]$. We obtain two results which show this relationship of duality between both concepts.

We establish the following notation. Given a quasinormed space $(E,\|\|)$ and two subspaces $E_{1}, E_{2} \subseteq E$ such that $E=E_{1} \oplus E_{2}$ is an algebraic direct sum, we denote the respective canonical projections by

$$
p_{k}:\left(E=E_{1} \oplus E_{2},\|\|\right) \rightarrow\left(E_{k},\|\|\right),
$$

which is defined by $x=x_{1}+x_{2} \mapsto x_{k}$, for each $k \in\{1,2\}$. If $E=E_{1} \oplus E_{2}$ is a topological direct sum, then the (topological) dual spaces also satisfy $E^{*}=E_{1}^{*} \oplus E_{2}^{*}$. 
In this case we denote the respective continuous canonical projections by

$$
p_{k *}:\left(E^{*}=E_{1}^{*} \oplus E_{2}^{*},\|\|_{*}\right) \rightarrow\left(E_{k}^{*},\|\|_{*}\right),
$$

defined by $f=f_{1}+f_{2} \mapsto f_{k}=\left.f\right|_{E_{k}}$ for every $k \in\{1,2\}$.

We also denote by $\delta_{i j}$ the Kronecker delta.

Our results are the following two theorems, which generalize Theorem 1.6 by Olevskii and Ulanovskii from the context of Hilbert spaces and $p=2$ to quasinormed spaces and $0<p \leq+\infty$.

Theorem 1.10. Let $(E,\|\|)$ be a quasinormed space. Let $\mathcal{S}=\left(e_{i}\right)_{i \in \mathbb{N}} \subseteq E$ and $\mathcal{S}^{*}=\left(\lambda_{i}\right)_{i \in \mathbb{N}} \subseteq E^{*}$ be sequences such that $\lambda_{i}\left(e_{j}\right)=\delta_{i j}$ for each $i, j \in \mathbb{N}$. Let $p \in$ $(0,+\infty]$, and let $J, L \subseteq \mathbb{N}$ be a partition of $\mathbb{N}$. Define $V:=\left(e_{j}\right)_{j \in J}, W:=\left(e_{l}\right)_{l \in L}$, $V^{*}:=\left(\lambda_{j}\right)_{j \in J}, W^{*}:=\left(\lambda_{l}\right)_{l \in L}$. Let $E_{1}, E_{2}$ be vector subspaces of $E$ such that $E=E_{1} \oplus E_{2}$ is an algebraic direct sum. Assume that $W$ is an upper $p$-frame* for $E$ and $p_{2}(V)$ is an upper $p$-frame* for $E_{2}$. Suppose that $p_{1}(V)$ is a lower $p$-frame* for $E_{1}$. Then:

(i) $p_{2 *}\left(W^{*}\right)$ is an upper $p$-Riesz sequence for $E_{2}^{*}$.

(ii) Suppose, in addition, that

(a) $W^{*}$ is a lower $p$-Riesz sequence for $E^{*}$.

(b) $E=E_{1} \oplus E_{2}$ is a topological direct sum.

Then $p_{2 *}\left(W^{*}\right)$ is a $p$-Riesz sequence for $E_{2}^{*}$.

We have a particular case of Theorem 1.10 when the sequence $\mathcal{S}=\left(e_{i}\right)_{i \in \mathbb{N}}$ is a normalized Schauder basis for $E$ and $\mathcal{S}^{*}=\left(\lambda_{i}\right)_{i \in \mathbb{N}}$ is the set of coordinate functionals associated to $\mathcal{S}$.

A reciprocal result of this theorem is the following one.

Theorem 1.11. Let $(E,\|\|)$ be a quasinormed space. Let $\mathcal{B}=\left(e_{i}\right)_{i \in \mathbb{N}} \subseteq E$ and $\mathcal{B}^{*}=\left(\lambda_{i}\right)_{i \in \mathbb{N}} \subseteq E^{*}$ be sequences such that $\lambda_{i}\left(e_{j}\right)=\delta_{i j}$ for each $i, j \in \mathbb{N}$. Suppose that $\mathcal{B}^{*}$ is a generator set of $E^{*}$. Let $p \in(0,+\infty]$ and let $J, L \subseteq \mathbb{N}$ be a partition of $\mathbb{N}$. Define $V:=\left(e_{j}\right)_{j \in J}, W:=\left(e_{l}\right)_{l \in L}, V^{*}:=\left(\lambda_{j}\right)_{j \in J}$, and $W^{*}:=\left(\lambda_{l}\right)_{l \in L}$. Let $E_{1}, E_{2}$ be vector subspaces of $E$ such that $E=E_{1} \oplus E_{2}$ is a topological direct sum. Suppose that

(i) $\mathcal{B}^{*}$ is a lower $p$-Riesz sequence for $E^{*}$;

(ii) $p_{1}(V)=\left(p_{1}\left(e_{j}\right)\right)_{j \in J}$ is an upper $p$-frame* for $E_{1}$;

(iii) $p_{1 *}\left(\mathcal{B}^{*}\right)$ is a Schauder basis for $E_{1}^{*}$.

If $p_{2 *}\left(W^{*}\right)=\left(p_{2 *}\left(\lambda_{l}\right)\right)_{l \in L}$ is an upper $p$-Riesz sequence for $E_{2}^{*}$, then $p_{1}(V)=$ $\left(p_{1}\left(e_{j}\right)\right)_{j \in J}$ is a lower $p$-frame* for $E_{1}$, and therefore $p_{1}(V)$ is a $p$-frame* for $E_{1}$.

Observe that if $(E,\|\|)$ is a Banach space, $\mathcal{B}$ is a normalized Schauder basis of $E$, and $\mathcal{B}^{*}$ is the set of coordinate functionals associated to $\mathcal{B}$, then $\mathcal{B}^{*}$ is a Schauder basis of $E^{*}$ if and only if $\mathcal{B}$ is shrinking (see [10, p. 8]); in particular, if $E$ is reflexive, then $\mathcal{B}^{*}$ is a Schauder basis of $E^{*}$, and therefore it is a generator set for $E^{*}$. 


\section{Proofs of Theorems 1.10 and 1.11}

In this section we will prove the duality theorems 1.10 and 1.11

\subsection{Proof of Theorem $\mathbf{1 . 1 0}$.}

Proof. Let us consider the sampling operators

$$
S_{1}:\left(E^{*},\|\|_{*}\right) \rightarrow\left(l^{p}(L),\|\|_{p}\right),
$$

defined by $f \rightarrow\left(f\left(e_{l}\right)\right)_{l \in L}$, and

$$
S_{2}:\left(E_{2}^{*},\|\|_{*}\right) \rightarrow\left(l^{p}(J),\|\|_{p}\right),
$$

defined by $f \rightarrow\left(f\left(p_{2}\left(e_{j}\right)\right)\right)_{j \in J}$. We observe that

- $W$ is an upper $p$-frame* for $E$ if and only if $S_{1}$ is continuous.

- $p_{2}(V)$ is an upper $p$-frame* for $E_{2}$ if and only if $S_{2}$ is continuous.

Suppose that $p_{1}(V)$ is a lower $p$-frame* for $E_{1}$. There exists a constant $A>0$ such that we have the inequality

$$
A\|x\|_{*} \leq\left\|\left(x\left(p_{1}\left(e_{j}\right)\right)\right)_{j \in J}\right\|_{p} \text { for each } x \in E_{1}^{*} .
$$

(i) Let us see that $p_{2 *}\left(W^{*}\right)=\left(p_{2 *}\left(\lambda_{l}\right)\right)_{l \in L}$ is an upper $p$-Riesz sequence for $E_{2}^{*}$.

Take $R_{0} \in \mathfrak{P}_{0}(L), R_{0} \neq \emptyset .\left\{\lambda_{l}\right\}_{l \in R_{0}} \subseteq E^{*}$, and

$$
\lambda_{l}\left(e_{j}\right)=\delta_{i l}=0 \quad \text { for each } l \in R_{0}, j \in J \text {, because } R_{0} \cap J=\emptyset \text {. }
$$

That is,

$$
\lambda_{l}(v)=0 \quad \text { for every } l \in R_{0}, v \in V \text {. }
$$

Take $\left\{c_{l}\right\}_{l \in R_{0}} \subseteq \mathbb{C}$. We define $f:=\sum_{l \in R_{0}} c_{l} \lambda_{l} \in E^{*} \subseteq E_{1}^{*} \oplus E_{2}^{*}$. We shall split $f=f_{1}+f_{2} \in E_{1}^{*} \oplus E_{2}^{*}$, where $f_{1} \in E_{1}^{*}, f_{2} \in E_{2}^{*}$ are unique (in fact: $f_{1}=\left.f\right|_{E_{1}} \in E_{1}^{*}$, $\left.f_{2}=\left.f\right|_{E_{2}} \in E_{2}^{*}\right)$. Besides,

$$
f_{1}=\left.\sum_{l \in R_{0}} c_{l} \lambda_{l}\right|_{E_{1}}, \quad f_{2}=\left.\sum_{l \in R_{0}} c_{l} \lambda_{l}\right|_{E_{2}} .
$$

For each $l \in R_{0}$ we have that $f\left(e_{l}\right)=\sum_{r \in R_{0}} c_{r} \lambda_{r}\left(e_{l}\right)=\sum_{r \in R_{0}} c_{r} \delta_{l r}=c_{l}$, so that

$$
f=\sum_{l \in R_{0}} f\left(e_{l}\right) \lambda_{l} \in E^{*} .
$$

On the other hand, for all $v \in V$ we have: $f(v)=\sum_{l \in R_{0}} f\left(e_{l}\right) \lambda_{l}(v)=0$, which is equivalent to $f_{1}\left(p_{1}(v)\right)=-f_{2}\left(p_{2}(v)\right)$. Since $p_{1}(V)$ is a lower $p$-frame* for $E_{1}$, we have the next consequence:

$$
\left\|f_{1}\right\|_{*}^{p} \leq \frac{1}{A^{p}} \sum_{v \in V}\left|f_{1}\left(p_{1}(v)\right)\right|^{p}=\frac{1}{A^{p}} \sum_{v \in V}\left|f_{2}\left(p_{2}(v)\right)\right|^{p} \leq D\left\|f_{2}\right\|_{*}^{p},
$$

where the last inequality comes from the continuity of $S_{2}$. Then

$$
\begin{aligned}
\sum_{l \in R_{0}}\left|c_{l}\right|^{p} & =\sum_{l \in R_{0}}\left|f\left(e_{l}\right)\right|^{p} \leq \sum_{w \in W}|f(w)|^{p} \leq D_{1}\|f\|_{*}^{p} \\
& =D_{1}\left\|f_{1}+f_{2}\right\|_{*}^{p} \leq D_{2}\left(\left\|f_{1}\right\|_{*}^{p}+\left\|f_{2}\right\|_{*}^{p}\right) \leq D_{2} \cdot(D+1)\left\|f_{2}\right\|_{*}^{p} \\
& =D_{3}\left\|f_{2}\right\|_{*}^{p},
\end{aligned}
$$


where in the second inequality we have used that $S_{1}$ is continuous. Defining $D_{4}:=$ $\frac{1}{D_{3}^{1 / p}}$ we obtain

$$
D_{4}\left(\sum_{l \in R_{0}}\left|c_{l}\right|^{p}\right)^{1 / p} \leq\left\|f_{2}\right\|_{*}
$$

For $p=+\infty$ it is completely analogous:

$$
\begin{aligned}
\max _{l \in R_{0}}\left|c_{l}\right| & =\max _{l \in R_{0}}\left|f\left(e_{l}\right)\right| \leq \max _{w \in W}|f(w)| \leq D_{1}\|f\|_{*} \\
& =D_{1}\left\|f_{1}+f_{2}\right\|_{*} \leq D_{1}\left(\left\|f_{1}\right\|_{*}+\left\|f_{2}\right\|_{*}\right) \leq D_{1} \cdot(D+1)\left\|f_{2}\right\|_{*} \\
& =D_{2}\left\|f_{2}\right\|_{*} .
\end{aligned}
$$

Conclusion: $p_{2 *}\left(W^{*}\right)=\left(p_{2 *}\left(\lambda_{l}\right)\right)_{l \in L}$ is an upper $p$-Riesz sequence for $E_{2}^{*}$.

(ii) Let us prove now the second part of the theorem.

Assume in addition that $W^{*}$ is a lower $p$-Riesz sequence for $E^{*}$ and $E=E_{1} \oplus E_{2}$ is a topological direct sum. Let us see that $p_{2 *}\left(W^{*}\right)$ is a $p$-Riesz sequence for $E_{2}^{*}$. By the first item we know that it is an upper $p$-Riesz sequence for $E_{2}^{*}$; we just have to prove that it is also a lower one.

Since the direct sum $E=E_{1} \oplus E_{2}$ is topological, the respective projections are continuous. In the same way we did before, we have:

$$
\sum_{l \in R_{0}}\left|c_{l}\right|^{p} \leq D_{3}\left\|f_{2}\right\|_{*}^{p} \leq D_{4}\|f\|_{*}^{p} \leq D_{5} \sum_{l \in R_{0}}\left|c_{l}\right|^{p} .
$$

Hence,

$$
\left(D_{3}\right)^{-1 / p}\left(\sum_{l \in R_{0}}\left|c_{l}\right|^{p}\right)^{1 / p} \leq\left\|f_{2}\right\|_{*} \leq\left(D_{5}\right)^{1 / p}\left(\sum_{l \in R_{0}}\left|c_{l}\right|^{p}\right)^{1 / p} .
$$

For $p=+\infty$ it is completely analogous:

$$
\left(D_{3}\right)^{-1} \max _{l \in R_{0}}\left|c_{l}\right| \leq\left\|f_{2}\right\|_{*} \leq D_{4}\|f\|_{*} \leq D_{5} \max _{l \in R_{0}}\left|c_{l}\right| .
$$

Conclusion: $p_{2 *}\left(W^{*}\right)$ is a $p$-Riesz sequence for $E_{2}^{*}$.

\subsection{Proof of Theorem $\mathbf{1 . 1 1}$.}

Proof. Recalling the definitions 1.8 and 1.9 of $p$-Riesz sequence and $p$-dual frame (upper, lower or both), we have:

(i) $\mathcal{B}^{*}=\left(\lambda_{i}\right)_{i \in \mathbb{N}}$ being a lower $p$-Riesz sequence for $E^{*}$ means that there exists a constant $B>0$ such that for each $I_{0} \in \mathfrak{P}_{0}(\mathbb{N})$ and each $a=\left(a_{i}\right)_{i \in I_{0}} \in \mathbb{C}^{I_{0}}$ we have that $\left\|\sum_{i \in I_{0}} a_{i} \lambda_{i}\right\|_{*} \leq B\left\|\left(a_{i}\right)_{i \in I_{0}}\right\|_{p}$.

(ii) $p_{1}(V)=\left(p_{1}\left(e_{j}\right)\right)_{j \in J}$ being an upper $p$-frame* for $E_{1}$ means that there exists a constant $C>0$ satisfying

$$
\left\|\left(g\left(p_{1}\left(e_{j}\right)\right)\right)_{j \in J}\right\|_{p} \leq C\|g\|_{*} \text { for each } g \in E_{1}^{*} .
$$


Since $E^{*}=E_{1}^{*} \oplus E_{2}^{*}$ is a topological direct sum, the canonical projection

$$
p_{k *}:\left(E^{*}=E_{1}^{*} \oplus E_{2}^{*},\|\|_{*}\right) \rightarrow\left(E_{k}^{*},\|\|_{*}\right),
$$

defined by $f=f_{1}+f_{2} \mapsto f_{k}=\left.f\right|_{E_{k}}$, is continuous for every $k \in\{1,2\}$.

Suppose that $p_{2 *}\left(W^{*}\right)=\left(p_{2 *}\left(\lambda_{l}\right)\right)_{l \in L}$ is an upper $p$-Riesz sequence for $E_{2}^{*}$. Let us see that $p_{1}(V)=\left(p_{1}\left(e_{j}\right)\right)_{j \in J}$ is a lower $p$-frame* for $E_{1}$; in other words, there exists a constant $D>0$ such that each $g \in E_{1}^{*}$ satisfies the inequality $D\|g\|_{*} \leq\left\|\left(g\left(p_{1}\left(e_{j}\right)\right)\right)_{j \in J}\right\|_{p}$.

By hypothesis, $\mathcal{B}^{*}=\left(\lambda_{i}\right)_{i \in \mathbb{N}}$ is a generator set of $E^{*}\left(\supseteq E_{1}^{*}, E_{2}^{*}\right)$. We will prove the result in two steps.

Step 1. Let $\tilde{g} \in p_{1 *}\left(\operatorname{span}\left(\mathcal{B}^{*}\right)\right)=\operatorname{span}\left\{p_{1 *}\left(\mathcal{B}^{*}\right)\right\} \subseteq E_{1}^{*}$. There exists $I_{0} \in \mathfrak{P}_{0}(\mathbb{N})$ and coefficients $c=\left(c_{i}\right)_{i \in I_{0}} \in \mathbb{C}^{I_{0}}$ such that

$$
\tilde{g}=\sum_{i \in I_{0}} c_{i} p_{1 *}\left(\lambda_{i}\right) .
$$

Let us consider the function $g:=\tilde{g} \circ p_{1} \in E^{*}$, which is an extension of $\tilde{g}$ to $E$, and satisfies $\left.g\right|_{E_{1}}=\tilde{g},\left.g\right|_{E_{2}}=0$. A direct calculus shows that $\|\tilde{g}\|_{*} \leq\|g\|_{*}$. Since $\mathcal{B}^{*}=\left(\lambda_{i}\right)_{i \in \mathbb{N}}$ is a generator set of $E^{*}$, there exists a sequence $a_{g}=\left(a_{i}\right)_{i \in \mathbb{N}}$ in $\mathbb{C}$ such that

$$
g=\sum_{i \in \mathbb{N}} a_{i} \lambda_{i} \in E^{*}
$$

Since $\lambda_{i}\left(e_{j}\right)=\delta_{i j}$ for each $i, j \in \mathbb{N}$, we have $a_{i}=g\left(e_{i}\right)$ for each $i \in \mathbb{N}$ and thus

$$
g=\sum_{i \in \mathbb{N}} g\left(e_{i}\right) \lambda_{i} \in E^{*}
$$

Hence, $\tilde{g}=\left.g\right|_{E_{1}}=p_{1 *}(g)=\sum_{i \in \mathbb{N}} g\left(e_{i}\right) p_{1 *}\left(\lambda_{i}\right) \in E_{1}^{*}$.

The assumption (iii) in the theorem implies the uniqueness of the coefficients, so that we have $c_{i}=g\left(e_{i}\right)$ for each $i \in I_{0}$ and $g\left(e_{i}\right)=0$ for every $i \in \mathbb{N} \backslash I_{0}$. Therefore, $\tilde{g}=\sum_{i \in I_{0}} g\left(e_{i}\right) p_{1 *}\left(\lambda_{i}\right)$ and $g=\sum_{i \in I_{0}} g\left(e_{i}\right) \lambda_{i}$.

Define $J_{0}:=J \cap I_{0} \in \mathfrak{P}_{0}(J), L_{0}:=L \cap I_{0} \in \mathfrak{P}_{0}(L)$. We define now:

$$
\begin{aligned}
& g_{V^{*}}:=\sum_{j \in J_{0}} g\left(e_{j}\right) \lambda_{j} \in \operatorname{span}\left(V^{*}\right), \\
& g_{W^{*}}:=\sum_{l \in L_{0}} g\left(e_{l}\right) \lambda_{l} \in \operatorname{span}\left(W^{*}\right) .
\end{aligned}
$$

We have $g=g_{V^{*}}+g_{W^{*}}$.

Since $0=\left.g\right|_{E_{2}}=p_{2 *}(g)=p_{2 *}\left(g_{V^{*}}\right)+p_{2 *}\left(g_{W^{*}}\right)$, we have

$$
p_{2 *}\left(g_{V^{*}}\right)=-p_{2 *}\left(g_{W^{*}}\right) .
$$


Therefore

$$
\begin{aligned}
\left\|g_{W^{*}}\right\|_{*} & =\left\|\sum_{l \in L_{0}} g\left(e_{l}\right) \lambda_{l}\right\|_{*} \leq D_{1}\left(\sum_{l \in L_{0}}\left|g\left(e_{l}\right)\right|^{p}\right)^{1 / p} \\
& \leq D_{2}\left\|\sum_{l \in L_{0}} g\left(e_{l}\right) p_{2 *}\left(\lambda_{l}\right)\right\|_{*}=D_{2}\left\|p_{2 *}\left(g_{W^{*}}\right)\right\|_{*}=D_{2}\left\|-p_{2 *}\left(g_{V^{*}}\right)\right\|_{*} \\
& =D_{2}\left\|p_{2 *}\left(g_{V^{*}}\right)\right\|_{*} \leq D^{\prime}\left\|g_{V^{*}}\right\|_{*} .
\end{aligned}
$$

The first inequality comes from the assumption (i), whereas the second inequality follows from the fact that $p_{2 *}\left(W^{*}\right)$ is an upper $p$-Riesz sequence for $E_{2}^{*}$. Therefore $\left\|g_{W^{*}}\right\|_{*} \leq D^{\prime}\left\|g_{V^{*}}\right\|_{*}$, with $D^{\prime}>0$ independent of $g$. Thus

$$
\begin{aligned}
\|g\|_{*} & =\left\|g_{V^{*}}+g_{W^{*}}\right\|_{*} \leq\left\|g_{V^{*}}\right\|_{*}+\left\|g_{W^{*}}\right\|_{*} \leq\left(1+D^{\prime}\right)\left\|g_{V^{*}}\right\|_{*} \\
& =D_{3}\left\|\sum_{j \in J_{0}} g\left(e_{j}\right) \lambda_{j}\right\|_{*} \leq D_{4}\left(\sum_{j \in J_{0}}\left|g\left(e_{j}\right)\right|^{p}\right)^{1 / p},
\end{aligned}
$$

where we have defined $D_{3}:=1+D^{\prime}, D_{4}:=D_{3} \cdot D_{1}$, and the last inequality is true by the assumption (i). So we have

$$
\|g\|_{*} \leq D_{4}\left(\sum_{j \in J_{0}}\left|g\left(e_{j}\right)\right|^{p}\right)^{1 / p}
$$

with $D_{4}>0$ independent of $g$. By definition of $g$ we also have that

$$
g\left(e_{j}\right)=\tilde{g}\left(p_{1}\left(e_{j}\right)\right) \quad \text { for each } j \in J_{0} .
$$

Then,

$$
\|\tilde{g}\|_{*} \leq\|g\|_{*} \leq D_{4}\left(\sum_{j \in J_{0}}\left|\tilde{g}\left(p_{1}\left(e_{j}\right)\right)\right|^{p}\right)^{1 / p} .
$$

We define $D:=\frac{1}{D_{4}}>0$. Then,

$$
D\|\tilde{g}\|_{*} \leq\left(\sum_{j \in J_{0}}\left|\tilde{g}\left(p_{1}\left(e_{j}\right)\right)\right|^{p}\right)^{1 / p} \leq\left(\sum_{j \in J}\left|\tilde{g}\left(p_{1}\left(e_{j}\right)\right)\right|^{p}\right)^{1 / p} .
$$

Therefore

$$
D\|\tilde{g}\|_{*} \leq\left(\sum_{j \in J}\left|\tilde{g}\left(p_{1}\left(e_{j}\right)\right)\right|^{p}\right)^{1 / p} .
$$

The assumption (iii) implies that $p_{1 *}\left(B^{*}\right) \subseteq E_{1}^{*}$ is a total set for $E_{1}^{*}$ :

$$
\overline{\operatorname{span}\left(p_{1 *}\left(B^{*}\right)\right)}=E_{1}^{*} \text {. }
$$

Define $S_{1 *}:=p_{1 *}\left(B^{*}\right)=\left\{p_{1 *}\left(\lambda_{i}\right): i \in \mathbb{N}\right\} \subseteq E_{1}^{*}$. We have proved that there exists a constant $D>0$ satisfying

$$
D\|\tilde{g}\|_{*} \leq\left(\sum_{j \in J}\left|\tilde{g}\left(p_{1}\left(e_{j}\right)\right)\right|^{p}\right)^{1 / p}=\left\|\left(\tilde{g}\left(p_{1}\left(e_{j}\right)\right)\right)_{j \in J}\right\|_{p}
$$

for all $\tilde{g} \in \operatorname{span}\left(S_{1}^{*}\right) \subseteq E_{1}^{*}$. 
For $p=+\infty$ we obtain the same result with standard modifications to the proof given above.

Step 2. General case: Let $g \in E_{1}^{*}=\overline{\operatorname{span}\left(S_{1}^{*}\right)}$. There exists a sequence $\left(g_{n}\right)_{n \in \mathbb{Z}^{+}} \in$ $\operatorname{span}\left(S_{1}^{*}\right) \subseteq E_{1}^{*}$ which converges to $g$ in $E_{1}^{*}$. By Step 1 we have that

$$
D\left\|g_{n}\right\|_{*} \leq\left\|\left(g_{n}\left(p_{1}\left(e_{j}\right)\right)\right)_{j \in J}\right\|_{p}
$$

for each $n \in \mathbb{Z}^{+}$. By the assumption (ii) we also have that

$$
\left\|\left(f\left(p_{1}\left(e_{j}\right)\right)\right)_{j \in J}\right\|_{p} \leq C_{3}\|f\|_{*} \text { for all } f \in E_{1}^{*} .
$$

In other words, the function

$$
\varphi:\left(E_{1}^{*},\|\|_{*}\right) \rightarrow\left(l^{p}(J),\|\|_{p}\right)
$$

given by

$$
f \rightarrow\left(f\left(p_{1}\left(e_{j}\right)\right)\right)_{j \in J}
$$

is well defined and continuous. Since $\left(g_{n}\right)_{n \in \mathbb{Z}^{+}}$converges to $g$ in $E_{1}^{*}$,

$$
\left(g_{n}\left(p_{1}\left(e_{j}\right)\right)\right)_{j \in J} \rightarrow_{n \rightarrow+\infty}\left(g\left(p_{1}\left(e_{j}\right)\right)\right)_{j \in J} \text { in }\left(l^{p}(J),\|\|_{p}\right),
$$

and thus

$$
\left\|\left(g_{n}\left(p_{1}\left(e_{j}\right)\right)\right)_{j \in J}\right\|_{p} \rightarrow_{n \rightarrow+\infty}\left\|\left(g\left(p_{1}\left(e_{j}\right)\right)\right)_{j \in J}\right\|_{p} .
$$

On the other hand, because of the convergence of $\left(g_{n}\right)_{n \in \mathbb{Z}^{+}}$to $g$, we have that

$$
\left\|g_{n}\right\|_{*} \rightarrow_{n \rightarrow+\infty}\|g\|_{*} \text {. }
$$

We saw before that $D\left\|g_{n}\right\|_{*} \leq\left\|\left(g_{n}\left(p_{1}\left(e_{j}\right)\right)\right)_{j \in J}\right\|_{p}$ for each $n \in \mathbb{Z}^{+}$. Taking limits when $n \rightarrow+\infty$, we obtain

$$
D\|g\|_{*} \leq\left\|\left(g\left(p_{1}\left(e_{j}\right)\right)\right)_{j \in J}\right\|_{p}
$$

where $D>0$ is independent of $g \in E_{1}^{*}$.

Conclusion: $p_{1}(V)=\left(p_{1}\left(e_{j}\right)\right)_{j \in J}$ is a $p$-frame* for $E_{1}$.

\section{ACKNowledgment}

The author would like to express his gratitude to the University of Murcia for its institutional support.

\section{REFERENCES}

[1] A. Aldroubi, Q. Sun and W.-S. Tang, Connection between $p$-frames and $p$-Riesz bases in locally finite SIS of $L^{p}(\mathbb{R})$, in Wavelet Applications in Signal and Image Processing VIII, 668-674, Proc. SPIE 4119, 2000. https://doi.org/10.1117/12.408656

[2] A. Aldroubi, Q. Sun and W.-S. Tang, $p$-frames and shift invariant subspaces of $L^{p}$, J. Fourier Anal. Appl. 7 (2001), no. 1, 1-21. MR 1812992

[3] P. Casazza, D. Han and D. Larson, Frames for Banach spaces, in The functional and harmonic analysis of wavelets and frames (San Antonio, TX, 1999), 149-182, Contemp. Math., 247, American Mathematical Society, Providence, RI, 1999. MR 1738089

[4] P. Casazza, O. Christensen and D. T. Stoeva, Frame expansions in separable Banach spaces, J. Math. Anal. Appl. 307 (2005), no. 2, 710-723. MR 2142455

[5] O. Christensen and D. T. Stoeva, $p$-frames in separable Banach spaces, Adv. Comput. Math. 18 (2003), no. 2-4, 117-126. MR 1968115 
[6] I. Daubechies, A. Grossmann and Y. Meyer, Painless nonorthogonal expansions, J. Math. Phys. 27 (1986), no. 5, 1271-1283. MR 0836025.

[7] R. J. Duffin and A. C. Schaeffer, A class of nonharmonic Fourier series, Trans. Amer. Math. Soc. 72 (1952), 341-366. MR 0047179.

[8] D. Gabor, Theory of communication, J. Inst. Electr. Eng. Part III Radio Commun. Eng. 93 (1946), no. 26, 429-441. https://doi.org/10.1049/ji-3-2.1946.0074

[9] K. Gröchenig, Describing functions: atomic decompositions versus frames, Monatsh. Math. 112 (1991), no. 1, 1-42. MR 1122103

[10] J. Lindenstrauss and L. Tzafriri, Classical Banach spaces. I, Springer-Verlag, Berlin, 1977. MR 0500056

[11] A. Olevskii and A. Ulanovskii, On the duality between sampling and interpolation, Anal. Math. 42 (2016), no. 1, 43-53. MR 3477355

[12] A. Olevskii and A. Ulanovskii, Functions with disconnected spectrum, University Lecture Series, 65, American Mathematical Society, Providence, RI, 2016. MR 3468930

[13] D. T. Stoeva, $X_{d}$-frames in Banach spaces and their duals, Int. J. Pure Appl. Math. 52 (2009), no. 1, 1-14. MR 2515188.

José Alfonso López Nicolás

Consejería de Educación, Cultura y Universidades de la Región de Murcia,

Avenida de la Fama, 15, C.P. 30003, Murcia, España

jaln4@um.es, josealfonso.lopez@murciaeduca.es

Received: September 30, 2018

Accepted: April 11, 2019 